\title{
Review
}

Digestion

\section{Medication Management of Irritable Bowel Syndrome}

\author{
Katy E. Trinkley ${ }^{a}$ Milap C. Nahata ${ }^{b}$ \\ a University of Colorado, Skaggs School of Pharmacy and Pharmaceutical Sciences, Aurora, Colo., and \\ ${ }^{\mathrm{b}}$ The Ohio State University Colleges of Pharmacy and Medicine, Columbus, Ohio, USA
}

\section{Key Words}

Irritable bowel syndrome - Medications - Therapeutics .

Functional gastrointestinal disorders · Constipation .

Diarrhea

\begin{abstract}
Background: Irritable bowel syndrome (IBS) is a complex syndrome that is difficult to manage. Here we present the evidence supporting medication treatments for specific IBS symptoms, discuss evidence-based management of IBS with medications including dose regimens and adverse effects and review progress on research for new IBS treatments. Summary: Currently, there is evidence to support improvements in specific IBS symptoms following treatment with loperamide, psyllium, bran, lubiprostone, linaclotide, amitriptyline, trimipramine, desipramine, citalopram, fluoxetine, paroxetine, dicyclomine, peppermint oil, rifaximin, ketotifen, pregabalin, gabapentin and octreotide and there are many new medications being investigated for the treatment of IBS. Key Message: Of the medications with demonstrated improvements for IBS symptoms, rifaximin, lubiprostone, linaclotide, fiber supplementation and peppermint oil have the most reliable evidence supporting their use for the treatment of IBS. Onset of efficacy for the various medications has
\end{abstract}

been noted to be as early as 6 days after initiation; however, the efficacy of most medications was not assessed prospectively at predefined periods. Additional studies of currently available and new medications are ongoing and are needed to better define their place in therapy and expand therapeutic options for the treatment of IBS. The most promising new medications for IBS include a variety of novel pharmacologic approaches, most notably the dual $\mu$-opioid receptor agonist and $\delta$-opioid antagonist, JNJ-27018966.

(C) 2014 S. Karger AG, Basel

\section{Background}

Irritable bowel syndrome (IBS) is a functional gastrointestinal disorder that affects nearly $15 \%$ of the US population [1]. It presents as a constellation of symptoms with marked inter-individual variability. IBS is categorized into three main types, which are constipation-predominant IBS (IBS-C), diarrhea-predominant IBS (IBSD) and mixed-IBS (IBS-M). While patients with IBS-C or

K.E. Trinkley and M.C. Nahata contributed equally to the writing of this work.

\section{KARGER}

E-Mail karger@karger.com www.karger.com/dig (c) 2014 S. Karger AG, Base

0012-2823/14/0894-0253\$39.50/0
Assist. Prof. Katy E. Trinkley, PharmD

Department of Clinical Pharmacy, Skaggs School of Pharmacy and Pharmaceutical Sciences, University of Colorado Anschutz Medical Campus 12850 E Montview Blvd, Mail Stop C238, Aurora, CO 80045 (USA)

E-Mail katy.trinkley@ucdenver.edu 
IBS-D experience constipation or diarrhea, respectively, most days, persons with IBS-M may experience both constipation and diarrhea. Regardless of IBS type, each is often associated with additional symptoms, which may include flatulence, feeling of incomplete evacuation, or abdominal pain. As a consequence of the burden of IBS, patients with IBS may have a decreased quality of life and productivity [1].

Treatment of IBS can improve quality of life and prevent lost productivity. Since there is no cure for IBS, treatment is targeted to alleviate the specific symptom(s). Treatment options for IBS include dietary and lifestyle modifications, as well as psychological and medication therapies. While there are many treatment options for IBS, there is no clear first-line treatment for all persons with IBS, because of the variable presentation of IBS and the limited evidence supporting efficacy of many of the treatment options. The challenge of choosing one treatment modality over another is often the first of many when managing IBS. If medications are the treatment modality chosen, the choice of medication is the next challenge, followed by optimally using the medication in patients.

When choosing a medication for a given patient with IBS, the medication chosen should have demonstrated efficacy for that patient's specific symptom(s), including IBS type (IBS-C, IBS-D, IBS-M). Deciding on a given medication requires an understanding of the evidence to date, which as mentioned earlier, is limited. Beyond the challenge of choosing the best medication for a given patient's symptom(s), clinicians are faced with the final challenge of managing the medication to optimize treatment response and safety in patients. While many of the medications to treat IBS are used for indications other than IBS, the dosing regimens and onset of efficacy for the medications are often different when treating IBS compared to other conditions. Optimizing medication therapies should improve the probability patients will benefit from treatment.

The purpose of this review is to present the evidence supporting medication treatments for specific IBS symptoms and discuss evidence-based management of medications for the treatment of IBS, including dose regimens, efficacy, and adverse effects in patients. While many of the medications reviewed here are extensively used in patient care, a detailed review of optimal medication therapy including mechanism of action, dosage requirement, efficacy and adverse effects has not been published. Further, this review summarizes potential emerging therapies for the treatment of IBS.

\section{Methods}

To review the evidence supporting the use of currently available medications for the treatment of IBS, a Medline search was conducting from 1973 until September 2013 using the following search terms: 'irritable bowel syndrome', 'therapeutics', 'antidiarrheal', 'laxatives', 'loperamide', 'dietary fiber', 'psyllium', 'calcium polycarbophil', 'methylcellulose', 'bulking agents', 'lubiprostone', 'linaclotide', 'tricyclic antidepressive agents' and its representative agents, 'serotonin reuptake inhibitors' and its representative agents, 'dicyclomine', 'hyoscyamine', 'hyoscine', 'peppermint oil', 'parasympatholytics', 'rifaximin', 'pregabalin', 'gabapentin', 'clonidine', 'octreotide', and 'ketotifen'.

All placebo-controlled trials assessing the efficacy of a medication for the treatment of the specific IBS symptoms of abdominal pain, bloating, stool form or consistency, frequency of bowel movements, presence of mucus upon defecation, bowel urgency, feelings of incomplete evacuation, flatulence, or borborygmi were included. In addition, studies assessing global improvement of IBS were included. IBS symptoms assessed as combined endpoints were not included. Studies were excluded if they were not available in the English language. Further exclusions included drugs not available in the USA, drugs available only through restricted access programs, and probiotics. Probiotics were excluded because of the wide variety of bacteria present in these products and the inconsistencies among various products.

All studies were critically evaluated for the strength of evidence supporting the efficacy of each medication for explicit IBS symptoms, similar to the criteria used by the American College of Gastroenterology and as described in table $1[2,3]$. Further, the studies were critically evaluated to determine the optimal management of each medication for the treatment of IBS, including careful assessment for safe and efficacious dosing regimens, onset of efficacy, duration of effect, and adverse effects. When applicable, additional resources, including FDA product labeling, were referenced to identify additional medication management issues that were not addressed in the trial(s).

To identify emerging therapies for and progress on the treatment of IBS, clinicaltrials.gov was queried, using the search terms 'irritable bowel syndrome' and 'IBS'. All trials registered that were ongoing or completed that included a medication as an intervention for IBS treatment were reviewed. Trials registered with medications that were no longer being investigated for the treatment of IBS were not reviewed, nor were trials of dietary supplements.

\section{Results and Discussions}

\section{Medications Currently Available for the Treatment of Irritable Bowel Syndrome}

The literature search resulted in 43 studies meeting the inclusion criteria that assessed the efficacy of medications for the treatment of IBS. Table 2 describes the studies that met inclusion criteria and their respective outcomes, whereas table 3 describes the overall efficacy of each medication by IBS type and symptom. With few exceptions, 
Table 1. Criteria used to evaluate the quality and strength of evidence in studies of medications for IBS. Adapted from the American College of Gastroenterology [3]

\begin{tabular}{lll}
\hline Level of evidence & Benefit vs. risk & Methodological quality of evidence \\
\hline 1A High-quality & Benefits clearly outweigh risk & RCT without important limitations \\
1B Moderate-quality & Benefits clearly outweigh risk & $\begin{array}{l}\text { RCT with important limitations (inconsistent results, } \\
\text { methodological flaws, indirect, or imprecise) }\end{array}$ \\
\hline
\end{tabular}

many of the studies were limited by short treatment duration and follow-up, imprecise or variable methods of identifying IBS and subtype, and small sample sizes. Medications with the most robust evidence supporting their use for the treatment of IBS were lubiprostone, linaclotide, rifaximin, fiber supplementation and peppermint oil. Table 4 describes the proposed mechanism of action of the medications for treating IBS.

Medication management issues that were often not addressed in the clinical trials included onset of efficacy and adverse effects. Many of the clinical trials inconsistently reported medication-related adverse effects and IBS symptom relief was only assessed at specific time points, making it hard to assess medication safety and determine the true onset of efficacy for medications. As a result, and as indicated below, other drug information references, most commonly FDA product labeling, were used to make recommendations on the desired medication-specific management of IBS.

\section{Lubiprostone}

Lubiprostone is a novel agent, FDA-approved for the treatment of IBS-C. The FDA-approved dose of lubiprostone for IBS-C is $8 \mu \mathrm{g}$ twice daily [4]. However, lubiprostone doses of $8,16,24$ and $48 \mu \mathrm{g}$ twice daily have been found to be effective and safe for most symptoms of IBS, with only $24 \mu \mathrm{g}$ twice daily consistently demonstrating efficacy for all symptoms studied [5]. If symptom improvement is not satisfactory with $8 \mu \mathrm{g}$ twice daily, a trial of higher doses may be reasonable. Onset of efficacy was apparent beginning at 1 month of treatment, but some symptoms were not improved until month 2 of treatment [5]. These data suggest patients should continue lubiprostone therapy for at least 1 month before discontinuing due to lack of efficacy. Adverse effects associated with lubiprostone included nausea, vomiting, diarrhea, flatulence, abdominal pain and distension, which were doserelated [5]. Administration with food and water or decreasing the dose may improve tolerability of lubiprostone.

Treatment of Irritable Bowel Syndrome
Given the clear evidence supporting the use, lubiprostone appears as a viable and preferred option for the treatment of IBS-C. Limiting lubiprostone's use are its high cost and lack of long-term studies.

\section{Linaclotide}

Another novel treatment and the newest FDA-approved drug for IBS-C is linaclotide. The FDA-approved dose of linaclotide is $290 \mu \mathrm{g}$ once daily. While 75 and 150 $\mu \mathrm{g}$ once daily were proven efficacious for some IBS symptoms and $600 \mu \mathrm{g}$ once daily was found effective in a dosefinding study, $300 \mu \mathrm{g}$ once daily was efficacious for all symptoms studied and was better tolerated than $600 \mu \mathrm{g}$ daily [6]. Linaclotide at $290 \mu \mathrm{g}$ once daily consistently improved IBS symptoms and was well tolerated [7-9]. Linaclotide is recommended to be taken on an empty stomach $30 \mathrm{~min}$ before breakfast to improve efficacy. Onset of symptom relief has occurred as early as week $1[6,7]$, which is a significant advantage when compared to most other IBS treatments. Diarrhea [6-9], flatulence $[6,8,9]$ and abdominal pain $[6,8,9]$ were the most common adverse effects associated with linaclotide. Diarrhea appeared to be dose-related [6] and most commonly occurred within the first 4 weeks of treatment [9].

Given the robust evidence supporting the use of linaclotide, linaclotide is a viable option for the treatment of IBS-C. Limitations of linaclotide's use are its high cost and lack of long-term studies.

\section{Rifaximin}

Rifaximin has been approved by the FDA for travelers' diarrhea and hepatic encephalopathy, but not for IBS. It has been approved, however, for IBS in many other countries. Some high-quality studies demonstrated its efficacy for IBS. The doses of rifaximin studied included $400 \mathrm{mg}$ three times daily, $550 \mathrm{mg}$ twice daily, and $550 \mathrm{mg}$ three times daily. Advantages of rifaximin were that the starting doses did not require titration and the treatment duration was limited to 10-14 days. After the short treatment period, the efficacy of rifaximin was observed to persist up 
Table 2. Placebo-controlled trials assessing the efficacy of medications for the treatment of IBS symptoms

\begin{tabular}{lllll}
\hline Medication & $\begin{array}{l}\text { Patients and } \\
\text { IBS subtype }\end{array}$ & Dose and treatment duration & Study design & $\begin{array}{l}\text { Level of } \\
\text { evidence }\end{array}$
\end{tabular}

Lubiprostone $\mathrm{n}=193 \quad$ 1) $8 \mu \mathrm{g}$ BID,

[5] IBS-C $16 \mu \mathrm{g} \mathrm{BID,} \mathrm{or}$

3) $24 \mu \mathrm{g}$ BID for 12 weeks
RCT double-blind; $1 \mathrm{~A}$

2 weeks' follow-up

Global improvement: significant improvement with $24 \mu \mathrm{g}$

\section{BID}

Abdominal pain: significant improvement

Bloating: significant improvement

Consistency: significant improvement with $24 \mu \mathrm{g}$ BID

Frequency: significant improvement

\begin{tabular}{lllll}
\hline $\begin{array}{l}\text { Lubiprostone } \\
\text { [48] }\end{array}$ & $\begin{array}{l}\mathrm{n}=62 \\
\text { IBS-C }\end{array}$ & $48 \mu \mathrm{g}$ QDay for 2 weeks & $\begin{array}{l}\text { RCT double-blind } \\
\text { crossover; } \\
\text { 2 weeks' washout }\end{array}$ & $\begin{array}{l}\text { Abdominal pain, frequency: no significant improvement } \\
\text { Consistency: significant improvement }\end{array}$ \\
\hline $\begin{array}{l}\text { Linaclotide } \\
\text { [7] }\end{array}$ & $\begin{array}{l}\mathrm{n}=1,604 \\
\text { IBS-C }\end{array}$ & $290 \mu \mathrm{g}$ QDay for 12 weeks & RCT double-blind $1 \mathrm{~A}$ & $\begin{array}{l}\text { Global improvement, abdominal pain, bloating: significant } \\
\text { improvement }\end{array}$ \\
\hline $\begin{array}{l}\text { Linaclotide } \\
{[8]}\end{array}$ & $\mathrm{n}=800$ & $290 \mu \mathrm{g}$ QDay for 12 weeks & $\begin{array}{l}\text { RCT double-blind; 1A } \\
4 \text { weeks' follow-up }\end{array}$ & $\begin{array}{l}\text { Abdominal pain, frequency, consistency, flatulence: } \\
\text { significant improvement }\end{array}$
\end{tabular}

in which the

linaclotide group

was randomized to

linaclotide or

placebo

\begin{tabular}{lllll}
\hline $\begin{array}{l}\text { Linaclotide } \\
{[9]}\end{array}$ & $\mathrm{n}=804$ & $290 \mu \mathrm{g}$ QDay for 12 weeks & RCT double-blind $1 \mathrm{~A}$ & $\begin{array}{l}\text { Global improvement, abdominal pain, frequency: significant } \\
\text { improvement }\end{array}$ \\
\hline Linaclotide & $\mathrm{n}=420$ & 1) $75 \mu$ g QDay, & RCT double-blind; $1 \mathrm{~A}$ & Global improvement: significant improvement with 150,
\end{tabular}

[6] IBS-C 2) $150 \mu \mathrm{g}$ QDay, 2 weeks' follow-up

3) $300 \mu \mathrm{g}$ QDay, or

300, and $600 \mu \mathrm{g}$ QDay

Abdominal pain, consistency, frequency: significant

improvement

Bloating: significant improvement with 75,300 and $600 \mu \mathrm{g}$ QDay

\begin{tabular}{|c|c|c|c|c|}
\hline Rifaximin [49] & $\begin{array}{l}\mathrm{n}=87 \\
\text { IBS-All }\end{array}$ & $\begin{array}{l}400 \mathrm{mg} \text { TID } \times 10 \text { days } \\
\text { for } 10 \text { days }\end{array}$ & $\begin{array}{l}\text { RCT double-blind } 1 \mathrm{~A} \\
\text { placebo; } \\
10 \text { weeks' follow-up }\end{array}$ & Bloating: significant improvement \\
\hline Rifaximin [10] & $\begin{array}{l}\mathrm{n}=388 \\
\text { IBS-D }\end{array}$ & $\begin{array}{l}550 \mathrm{mg} \text { BID } \times 14 \text { days } \\
\text { for } 14 \text { days }\end{array}$ & $\begin{array}{l}\text { RCT double-blind; } 1 \mathrm{~A} \\
12 \text { weeks' follow-up }\end{array}$ & Bloating: significant improvement \\
\hline Rifaximin [50] & $\begin{array}{l}\mathrm{n}=125 \\
\text { IBS-D and } \\
\text { mixed }\end{array}$ & $550 \mathrm{mg}$ TID for 2 weeks & $\begin{array}{l}\text { RCT double-blind; } 1 \mathrm{~A} \\
10 \text { weeks' follow-up }\end{array}$ & $\begin{array}{l}\text { Global improvement, abdominal pain, bloating, consistency: } \\
\text { significant improvement }\end{array}$ \\
\hline Psyllium [15] & $\begin{array}{l}\mathrm{n}=80 \\
\text { IBS-All }\end{array}$ & $3.6 \mathrm{~g}$ sachet TID for 12 weeks & RCT double-blind $1 \mathrm{~B}$ & $\begin{array}{l}\text { Global improvement: significant improvement } \\
\text { Frequency: significant improvement }\end{array}$ \\
\hline Psyllium [13] & $\begin{array}{l}\mathrm{n}=20 \\
\text { IBS-All }\end{array}$ & $30 \mathrm{~g}$ QDay for 4 weeks & $\begin{array}{l}\text { RCT double-blind } 1 \mathrm{~B} \\
\text { crossover; } \\
7-10 \text { days' washout }\end{array}$ & $\begin{array}{l}\text { Global improvement: significant improvement } \\
\text { Frequency: no significant improvement }\end{array}$ \\
\hline Psyllium [16] & $\begin{array}{l}\mathrm{n}=80 \\
\text { IBS-All }\end{array}$ & $3 \mathrm{~g}$ sachet BID for 4 weeks & RCT double-blind 1B & Global improvement: no significant improvement \\
\hline Psyllium [17] & $\begin{array}{l}\mathrm{n}=12 \\
\text { IBS-All }\end{array}$ & $3.5 \mathrm{~g}$ BID for 16 weeks & RCT double-blind $1 \mathrm{~B}$ & Global improvement: significant improvement \\
\hline Psyllium [18] & $\begin{array}{l}\mathrm{n}=77 \\
\text { IBS-All }\end{array}$ & $6.4 \mathrm{~g}$ TID for 8 weeks & RCT double-blind $1 \mathrm{~B}$ & $\begin{array}{l}\text { Global improvement, abdominal pain, consistency: no } \\
\text { significant improvement }\end{array}$ \\
\hline $\begin{array}{l}\text { Psyllium or } \\
\text { Bran [14] }\end{array}$ & $\begin{array}{l}\mathrm{n}=275 \\
\text { IBS-All }\end{array}$ & $\begin{array}{l}\text { 1) } 5 \text { g psyllium, BID, or } \\
\text { 2) } 5 \text { g bran BID for } 12 \text { weeks }\end{array}$ & RCT double-blind $1 \mathrm{~A}$ & $\begin{array}{l}\text { Global improvement: psyllium - significant; bran - no } \\
\text { improvement } \\
\text { Abdominal pain: psyllium - significant improvement; } \\
\text { bran - significant improvement }\end{array}$ \\
\hline $\begin{array}{l}\text { Peppermint } \\
\text { oil [21] }\end{array}$ & $\begin{array}{l}\mathrm{n}=50 \\
\text { IBS-All }\end{array}$ & $550 \mathrm{mg}$ QDay for 4 weeks & $\begin{array}{l}\text { RCT double-blind; } 1 \mathrm{~A} \\
4 \text { weeks' follow-up }\end{array}$ & $\begin{array}{l}\text { Global improvement, abdominal pain, bloating, } \\
\text { urgency, incomplete evacuation: significant improvement }\end{array}$ \\
\hline $\begin{array}{l}\text { Peppermint } \\
\text { oil [19] }\end{array}$ & $\begin{array}{l}\mathrm{n}=110 \\
\text { IBS-All }\end{array}$ & $\begin{array}{l}187 \mathrm{mg} \text { TID or QID for } \\
4 \text { weeks }\end{array}$ & RCT double-blind $1 \mathrm{~B}$ & $\begin{array}{l}\text { Abdominal pain, frequency, flatulence, borborygmi: } \\
\text { significant improvement }\end{array}$ \\
\hline $\begin{array}{l}\text { Peppermint } \\
\text { oil [20] }\end{array}$ & $\begin{array}{l}\mathrm{n}=74 \\
\text { IBS-All }\end{array}$ & $\begin{array}{l}187 \mathrm{mg}(2 \mathrm{ml}) \mathrm{TID} 30 \mathrm{~min} \\
\text { before meals for } 6 \text { weeks }\end{array}$ & RCT double-blind $1 \mathrm{~B}$ & $\begin{array}{l}\text { Abdominal pain: significant improvement } \\
\text { Bloating, consistency, frequency, urgency, incomplete } \\
\text { evacuation, flatulence: no significant improvement }\end{array}$ \\
\hline $\begin{array}{l}\text { Peppermint } \\
\text { oil [51] }\end{array}$ & $\begin{array}{l}\mathrm{n}=90 \\
\text { IBS-All }\end{array}$ & $\begin{array}{l}187 \mathrm{mg}(2 \mathrm{ml}) \text { TID } 30 \mathrm{~min} \\
\text { before meals for } 8 \text { weeks }\end{array}$ & RCT double-blind $1 \mathrm{~A}$ & $\begin{array}{l}\text { Abdominal pain: significant improvement } \\
\text { Consistency, frequency, flatulence: no significant } \\
\text { improvement }\end{array}$ \\
\hline $\begin{array}{l}\text { Hyoscine } \\
{[17]}\end{array}$ & $\begin{array}{l}\mathrm{n}=12 \\
\text { IBS-All }\end{array}$ & $10 \mathrm{mg}$ QID for 16 weeks & RCT double-blind $1 \mathrm{~B}$ & Global improvement: no significant improvement \\
\hline
\end{tabular}


Table 2 (continued)

\begin{tabular}{|c|c|c|c|c|c|}
\hline Medication & $\begin{array}{l}\text { Patients and } \\
\text { IBS subtype }\end{array}$ & Dose and treatment duration & Study design & $\begin{array}{l}\text { Level of } \\
\text { evidence }\end{array}$ & Clinical efficacy results by symptom(s) \\
\hline $\begin{array}{l}\text { Dicyclomine } \\
{[22]}\end{array}$ & $\begin{array}{l}\mathrm{n}=97 \\
\mathrm{IBS}-\mathrm{C}\end{array}$ & $40 \mathrm{mg}$ QID for 2 weeks & RCT double-blind & $1 \mathrm{~B}$ & $\begin{array}{l}\text { Global improvement, abdominal pain: significant } \\
\text { improvement }\end{array}$ \\
\hline $\begin{array}{l}\text { Loperamide } \\
{[52]}\end{array}$ & $\begin{array}{l}\mathrm{n}=60 \\
\text { IBS-All }\end{array}$ & $4 \mathrm{mg}$ QHS for 3 weeks & RCT double-blind & $1 \mathrm{~B}$ & $\begin{array}{l}\text { Global improvement, abdominal pain, consistency, } \\
\text { frequency: significant improvement }\end{array}$ \\
\hline $\begin{array}{l}\text { Loperamide } \\
\text { [23] }\end{array}$ & $\begin{array}{l}\mathrm{n}=28 \\
\text { IBS-All }\end{array}$ & $\begin{array}{l}2 \mathrm{mg} \text { BID, then titrate to } \\
\text { max. } 12 \mathrm{mg} \text { as tolerated for } \\
5 \text { weeks (mean dose } 4.8 \mathrm{mg} / \text { day) }\end{array}$ & $\begin{array}{l}\text { RCT double-blind } \\
\text { crossover }\end{array}$ & $1 \mathrm{~B}$ & $\begin{array}{l}\text { Consistency, frequency, urgency, borborygmi: significant } \\
\text { improvement }\end{array}$ \\
\hline $\begin{array}{l}\text { Loperamide } \\
{[24]}\end{array}$ & $\begin{array}{l}\mathrm{n}=90 \\
\text { IBS-All }\end{array}$ & $\begin{array}{l}2 \mathrm{mg} \text { QHS, then titrate } \\
\text { to max. } 6 \mathrm{mg} \text { for } 5 \text { weeks } \\
\text { as needed and tolerated } \\
\text { (mean dose } 3 \mathrm{mg} / \text { day) }\end{array}$ & RCT double-blind & $1 \mathrm{~A}$ & Consistency, frequency: significant improvement \\
\hline $\begin{array}{l}\text { Loperamide } \\
{[25]}\end{array}$ & $\begin{array}{l}\mathrm{n}=25 \\
\mathrm{IBS}-\mathrm{D}\end{array}$ & $\begin{array}{l}2 \mathrm{mg} \text { QHS, titrated Qweek } \\
\text { to max. } 8 \mathrm{mg} \text { QHS for } \\
13 \text { weeks as needed and } \\
\text { tolerated (mean dose } 4 \mathrm{mg} / \text { day) }\end{array}$ & RCT double-blind & $1 \mathrm{~B}$ & $\begin{array}{l}\text { Global improvement, abdominal pain, consistency, urgency: } \\
\text { significant improvement } \\
\text { Frequency, flatulence, borborygmi: no significant } \\
\text { improvement }\end{array}$ \\
\hline $\begin{array}{l}\text { Amitriptyline } \\
\text { [31] }\end{array}$ & $\begin{array}{l}\mathrm{n}=40 \\
\text { IBS-All }\end{array}$ & $\begin{array}{l}25 \mathrm{mg} \text { QHS } \times 1 \text { week } \\
\text { titrated to } 50 \mathrm{mg} \text { QHS } \times 1 \text { week, } \\
\text { then } 75 \mathrm{mg} \text { QHS for } 10 \text { weeks }\end{array}$ & RCT double-blind & $1 \mathrm{~B}$ & $\begin{array}{l}\text { Global improvement, abdominal pain: significant } \\
\text { improvement } \\
\text { Frequency, flatulence: no significant improvement }\end{array}$ \\
\hline $\begin{array}{l}\text { Amitriptyline } \\
{[32]}\end{array}$ & $\begin{array}{l}\mathrm{n}=33 \\
\text { IBS-All } \\
\text { adolescents } \\
\text { without } \\
\text { psychiatric } \\
\text { disorders }\end{array}$ & $\begin{array}{l}\text { 1) if } 30-50 \mathrm{~kg}: 10 \mathrm{mg} \text { QHS, } \\
\text { 2) if } 50-80 \mathrm{~kg}: 20 \mathrm{mg} \text { QHS, or } \\
\text { 3) if >80 kg: } 30 \mathrm{mg} \text { QHS for } \\
8 \text { weeks }\end{array}$ & $\begin{array}{l}\text { RCT double-blind; } \\
3 \text { weeks' follow-up }\end{array}$ & $1 \mathrm{~B}$ & $\begin{array}{l}\text { Global improvement, abdominal pain: significant } \\
\text { improvement }\end{array}$ \\
\hline $\begin{array}{l}\text { Amitriptyline } \\
{[30]}\end{array}$ & $\begin{array}{l}\mathrm{n}=54 \\
\mathrm{IBS}-\mathrm{D}\end{array}$ & $10 \mathrm{mg}$ QHS for 8 weeks & RCT double-blind & $1 \mathrm{~A}$ & $\begin{array}{l}\text { Global improvement, consistency, incomplete evacuation: } \\
\text { significant improvement } \\
\text { Abdominal pain, mucus, flatulence: no significant } \\
\text { improvement }\end{array}$ \\
\hline $\begin{array}{l}\text { Trimipramine } \\
{[33]}\end{array}$ & $\begin{array}{l}\mathrm{n}=61 \\
\text { IBS-All }\end{array}$ & $50 \mathrm{mg}$ QDay for 4 weeks & RCT double-blind & $1 \mathrm{~B}$ & Mucus: significant improvement \\
\hline Trimipramine & $\begin{array}{l}\mathrm{n}=428 \\
\text { IBS-All }\end{array}$ & $\begin{array}{l}\text { 1) } 50 \mathrm{mg} \text { QHS, } \\
\text { 2) } 10 \mathrm{mg} \text { QAM }+40 \mathrm{mg} Q P M \text {, } \\
\text { 3) } 35 \mathrm{mg} \text { QPM, or } \\
\text { 4) } 10 \mathrm{mg} \text { TID for } \geq 6 \text { weeks }\end{array}$ & RCT double-blind & $1 \mathrm{~B}$ & $\begin{array}{l}\text { Abdominal pain: significant improvement with } 50 \mathrm{mg} \text { QHS, } \\
\text { and } 10 \mathrm{mg} \text { QAM + } 40 \mathrm{mg} \mathrm{QPM} \\
\text { Frequency: no significant improvement }\end{array}$ \\
\hline $\begin{array}{l}\text { Imipramine } \\
\text { [53] }\end{array}$ & $\begin{array}{l}\mathrm{n}=51 \\
\text { IBS-All } \\
\text { without } \\
\text { psychiatric } \\
\text { disorders }\end{array}$ & $\begin{array}{l}25 \mathrm{mg} \text { QHS } \times 2 \text { weeks, then } \\
50 \mathrm{mg} \text { QHS for } 10 \text { weeks }\end{array}$ & RCT double-blind & $1 \mathrm{~A}$ & $\begin{array}{l}\text { Global improvement, abdominal pain, frequency: no } \\
\text { significant improvement }\end{array}$ \\
\hline $\begin{array}{l}\text { Desipramine } \\
{[35]}\end{array}$ & $\begin{array}{l}\mathrm{n}=28 \\
\text { IBS-All }\end{array}$ & $\begin{array}{l}50 \mathrm{mg} \text { QHS } \times 1 \text { week, } \\
100 \mathrm{mg} \text { QHS } \times 1 \text { week, then } \\
150 \mathrm{mg} \text { QHS for } 4 \text { weeks }\end{array}$ & $\begin{array}{l}\text { RCT double-blind } \\
\text { crossover; } \\
2 \text { weeks' washout }\end{array}$ & $1 \mathrm{~B}$ & $\begin{array}{l}\text { Global improvement: improvement, significance not } \\
\text { reported } \\
\text { Abdominal pain: significant improvement in IBS-D } \\
\text { subgroup } \\
\text { Frequency: significant improvement }\end{array}$ \\
\hline $\begin{array}{l}\text { Desipramine } \\
{[54]}\end{array}$ & $\begin{array}{l}\mathrm{n}=31 \\
\text { IBS-All with } \\
\text { depression }\end{array}$ & $150 \mathrm{mg}$ QHS for 6 weeks & RCT double-blind & $1 \mathrm{~B}$ & Abdominal pain: no significant improvement \\
\hline Doxepin [36] & $\begin{array}{l}\mathrm{n}=44 \\
\text { IBS-All }\end{array}$ & $75 \mathrm{mg}$ QHS for 6 weeks & $\begin{array}{l}\text { RCT double-blind; } \\
4 \text { weeks' follow-up }\end{array}$ & $1 \mathrm{~B}$ & $\begin{array}{l}\text { Global improvement, abdominal pain, incomplete } \\
\text { evacuation: significant improvement }\end{array}$ \\
\hline $\begin{array}{l}\text { Citalopram } \\
{[53]}\end{array}$ & $\begin{array}{l}\mathrm{n}=51 \\
\text { IBS-All } \\
\text { without } \\
\text { psychiatric } \\
\text { illness }\end{array}$ & $\begin{array}{l}20 \mathrm{mg} \text { QAM } \times 2 \text { weeks, then } \\
40 \mathrm{mg} \text { QAM for } 10 \text { weeks }\end{array}$ & RCT double-blind & $1 \mathrm{~A}$ & $\begin{array}{l}\text { Global improvement, abdominal pain, frequency: no } \\
\text { significant improvement }\end{array}$ \\
\hline $\begin{array}{l}\text { Citalopram } \\
{[19]}\end{array}$ & $\begin{array}{l}\mathrm{n}=23 \\
\text { IBS-All } \\
\text { without } \\
\text { depression }\end{array}$ & $\begin{array}{l}20 \mathrm{mg} \text { QDay } \times 3 \text { weeks, then } \\
40 \mathrm{mg} \text { QDay } \times 3 \text { weeks }\end{array}$ & $\begin{array}{l}\text { RCT crossover; } \\
3 \text { weeks' washout }\end{array}$ & $1 \mathrm{~B}$ & $\begin{array}{l}\text { Global improvement, abdominal pain, bloating, urgency, } \\
\text { incomplete evacuation: significant improvement }\end{array}$ \\
\hline $\begin{array}{l}\text { Citalopram } \\
{[55]}\end{array}$ & $\begin{array}{l}\mathrm{n}=54 \\
\text { IBS-All }\end{array}$ & $20 \mathrm{mg}$ QDay for 8 weeks & RCT double-blind & $1 \mathrm{~A}$ & Global improvement: no significant improvement \\
\hline
\end{tabular}


Table 2 (continued)

\begin{tabular}{|c|c|c|c|c|c|}
\hline Medication & $\begin{array}{l}\text { Patients and } \\
\text { IBS subtype }\end{array}$ & Dose and treatment duration & Study design & $\begin{array}{l}\text { Level of } \\
\text { evidence }\end{array}$ & Clinical efficacy results by symptom(s) \\
\hline $\begin{array}{l}\text { Fluoxetine } \\
{[56]}\end{array}$ & $\begin{array}{l}\mathrm{n}=40 \\
\text { IBS-All } \\
\text { without } \\
\text { depression }\end{array}$ & $20 \mathrm{mg}$ QHS for 6 weeks & RCT double-blind & $1 \mathrm{~B}$ & $\begin{array}{l}\text { Global improvement, abdominal pain, bloating, urgency, } \\
\text { incomplete evacuation, flatulence: } \\
\text { No significant improvement }\end{array}$ \\
\hline $\begin{array}{l}\text { Fluoxetine } \\
{[28]}\end{array}$ & $\begin{array}{l}\mathrm{n}=44 \\
\text { IBS-C }\end{array}$ & 20 mg QDay for 12 weeks & $\begin{array}{l}\text { RCT double-blind; } \\
4 \text { weeks' follow-up }\end{array}$ & $1 \mathrm{~A}$ & Bloating, consistency, frequency: significant improvement \\
\hline $\begin{array}{l}\text { Paroxetine } \\
\text { CR [29] }\end{array}$ & $\begin{array}{l}\mathrm{n}=72 \\
\text { IBS-All } \\
\text { without } \\
\text { psychiatric } \\
\text { disorders }\end{array}$ & $\begin{array}{l}12.5 \mathrm{mg} \text { titrated to } 50 \mathrm{mg} \\
\text { QDay as tolerated for } \\
2 \text { weeks (mean dose } 30 \mathrm{mg} / \text { day) }\end{array}$ & RCT double-blind & $1 \mathrm{~A}$ & $\begin{array}{l}\text { Global improvement: significant improvement } \\
\text { Abdominal pain: no improvement }\end{array}$ \\
\hline $\begin{array}{l}\text { Ketotifen } \\
{[37]}\end{array}$ & $\begin{array}{l}\mathrm{n}=60 \\
\text { IBS-All }\end{array}$ & $\begin{array}{l}2 \mathrm{mg} \text { BID } \times 2 \text { weeks, } \\
4 \mathrm{mg} \text { BID } \times 2 \text { weeks, then } \\
6 \mathrm{mg} \text { BID } \times 4 \text { weeks }\end{array}$ & $\begin{array}{l}\text { RCT double-blind; } \\
2 \text { weeks' follow-up }\end{array}$ & 1B & $\begin{array}{l}\text { Global improvement: no significant improvement } \\
\text { Abdominal pain, bloating, consistency, frequency, } \\
\text { incomplete evacuation, flatulence: significant improvement }\end{array}$ \\
\hline $\begin{array}{l}\text { Pregabalin } \\
\text { [39] }\end{array}$ & $\begin{array}{l}\mathrm{n}=26 \\
\text { IBS-All } \\
\text { without } \\
\text { psychiatric } \\
\text { disorders }\end{array}$ & $\begin{array}{l}50 \mathrm{mg} \text { TID } \times 3 \text { days titrated to } \\
100 \mathrm{mg} \text { TID } \times 4 \text { days, } \\
150 \mathrm{mg} \text { TID } \times 4 \text { days, then } \\
200 \mathrm{mg} \text { TID for } 13 \text { days }\end{array}$ & RCT double-blind & $1 \mathrm{~B}$ & Abdominal pain, urgency: significant improvement \\
\hline $\begin{array}{l}\text { Gabapentin } \\
{[38]}\end{array}$ & $\begin{array}{l}\mathrm{n}=40 \\
\text { IBS-D }\end{array}$ & $\begin{array}{l}100 \mathrm{mg} \text { TID } \times 3 \text { days titrated to } \\
200 \mathrm{mg} \text { TID } \times 2 \text { days }\end{array}$ & RCT double-blind & $1 \mathrm{~B}$ & Abdominal pain, bloating: significant improvement \\
\hline $\begin{array}{l}\text { Clonidine } \\
{[42]}\end{array}$ & $\begin{array}{l}\mathrm{n}=44 \\
\text { IBS-D }\end{array}$ & $0.1 \mathrm{mg}$ BID for 4 weeks & $\begin{array}{l}\text { RCT double-blind; } \\
\text { pilot study }\end{array}$ & $1 \mathrm{~B}$ & $\begin{array}{l}\text { Global improvement: significant improvement } \\
\text { Consistency, frequency: no significant improvement }\end{array}$ \\
\hline $\begin{array}{l}\text { Octreotide } \\
{[40]}\end{array}$ & $\begin{array}{l}\mathrm{n}=46 \\
\mathrm{IBS}-\mathrm{D} \text { and } \\
\mathrm{A}\end{array}$ & 20 mg IM Q4weeks for 8 weeks & RCT double-blind & $1 \mathrm{~B}$ & $\begin{array}{l}\text { Global improvement, abdominal pain, bloating, frequency, } \\
\text { flatulence, incomplete evacuation: no significant } \\
\text { improvement } \\
\text { Consistency: significant improvement }\end{array}$ \\
\hline
\end{tabular}

to 12 weeks [10]. Given the high cost of rifaximin, the short treatment duration makes treatment more feasible for many patients. Although the onset of efficacy is unclear, it may occur as early as 3 weeks. Adverse effects with rifaximin were no different than placebo in clinical trials, yet patients should be counseled on the potential for peripheral edema, dizziness, fatigue, and nausea [11]; however, the incidence of these adverse effects during short courses of treatment so far appears to be extremely low.

The favorable adverse effect profile of rifaximin in IBS trials in combination with the robust evidence supporting its efficacy suggest rifaximin may become a preferred option for the treatment of IBS. However, the manufacturer's supplemental new drug application for treating nonconstipation IBS has been rejected by the FDA. Additional data were needed by the FDA [12]. Limiting rifaximin are also its high cost and lack of long-term studies. The lasting duration of rifaximin's effect on IBS after treatment cessation is not yet well defined.

\section{Fiber Supplements}

Although fiber supplements are not regulated by the FDA, it has been found to be efficacious for a number of
IBS symptoms. Both psyllium and bran have been studied and found effective for treating some symptoms associated with IBS. Efficacious doses of psyllium ranged from $3 \mathrm{~g}$ twice daily to $6.4 \mathrm{~g}$ three times daily to $30 \mathrm{~g}$ once daily. Onset of efficacy with psyllium has occurred within 4 weeks of use $[13,14]$. At $10 \mathrm{~g}$ twice daily, bran was found to improve IBS symptoms, but the onset of efficacy was delayed until month 3 of treatment [14]. In clinical trials, psyllium and bran were well tolerated when initiated at the aforementioned maintenance doses, suggesting titration may not be necessary [13-18]; however, clinical experience with poor patient tolerance when no titration was performed suggests titration is important with fiber supplementation. Similarly, although adverse effects were found to be similar to placebo in clinical trials, patients were still at risk for adverse effects observed in persons without IBS, including abdominal pain, constipation, nausea, flatulence, and diarrhea.

There are several advantages to fiber supplementation for treating IBS, including its low cost, long-term experience with use, ease of access, and generally mild adverse effect profile. Further, the level of evidence supporting fiber supplementation has been greater than many other 
Table 3. Efficacy of medications for the treatment of specific IBS symptoms: clinical outcomes of placebo-controlled trials

\begin{tabular}{|c|c|c|c|c|c|c|c|c|c|c|}
\hline Medication & $\begin{array}{l}\text { Global } \\
\text { improvement }\end{array}$ & $\begin{array}{l}\text { Abdominal } \\
\text { pain }\end{array}$ & Bloating & Consistency & Frequency & Mucus & Urgency & $\begin{array}{l}\text { Incomplete } \\
\text { evacuation }\end{array}$ & Flatulence & Borborygmi \\
\hline Lubiprostone & $\mathrm{C}:+$ & C: +- & $\mathrm{C}:+$ & $\mathrm{C}:++$ & $\mathrm{C}:+-$ & & & & & \\
\hline Linaclotide & $\mathrm{C}:+++$ & $\mathrm{C}:++++$ & $\mathrm{C}:++$ & $\mathrm{C}:++$ & $\mathrm{C}:+++$ & & & & $\mathrm{C}:+$ & \\
\hline Rifaximin & $\mathrm{D}:+$ & $\mathrm{D}:+$ & $\begin{array}{l}\mathrm{A}:+ \\
\mathrm{D}:+ \\
\mathrm{D} \text { and } \mathrm{M}:+\end{array}$ & $\mathrm{D}:+$ & & & & & & \\
\hline Psyllium & A: +++-- & A: - & & A: - & A: +- & & & & & \\
\hline Bran & A: - & A: + & & & & & & & & \\
\hline Dicyclomine & C: - & & & & & & & & & \\
\hline Hyoscine & A: - & & & & & & & & & \\
\hline Peppermint oil & A: + & $\mathrm{A}:++++$ & A: +- & A: - - & A: +-- & & A: +- & A: +- & A: +-- & A: + \\
\hline Loperamide & $\mathrm{A}:++$ & $\mathrm{A}:++$ & & $\mathrm{A}:++++$ & $\mathrm{A}:+++-$ & & $\mathrm{A}:++$ & & A: - & $\mathrm{A}:+-$ \\
\hline Amitriptyline & $\begin{array}{l}\text { A: }++ \\
\mathrm{D}:+\end{array}$ & $\begin{array}{l}\text { A: }++ \\
\text { D: }-\end{array}$ & & $\mathrm{D}:+$ & A: - & D: - & & $\mathrm{D}:+$ & $\begin{array}{l}\text { A: - } \\
\text { D: - }\end{array}$ & \\
\hline Trimipramine & A: + & A: +- & & A: + & A: - & A: -+ & & A: + & A: - & \\
\hline Imipramine & A: - & A: - & & & A: - & & & & & \\
\hline Desipramine & A: + & $\begin{array}{l}\text { A: }- \\
\text { D: }+\end{array}$ & & & A: + & & & & & \\
\hline Doxepin & $\mathrm{A}:+$ & $\mathrm{A}:+$ & & & & & & A: + & & \\
\hline Citalopram & A: +-- & A: +- & A: + & & A: - & & A: + & A: + & & \\
\hline Fluoxetine & A: - & A: - & $\begin{array}{l}\text { A: }- \\
\text { C: }+\end{array}$ & C: + & C: + & & A: - & A: - & A: - & \\
\hline Paroxetine & A: + & A: - & & & & & & & & \\
\hline Ketotifen & A: - & A: + & A: + & A: + & A: + & & & A: + & A: + & \\
\hline Pregabalin & A: + & & & & & & A: + & & & \\
\hline Gabapentin & & D: + & D: + & & & & & & & \\
\hline Clonidine & $\mathrm{D}:+$ & & & & & & & & & \\
\hline Octreotide & A and D:- & A and D:- & A and D:- & A and D: + & A and D:- & & & A and D: - & A and D:- & \\
\hline
\end{tabular}

$A$ = Subject with all types of IBS were included; $C$ = subjects included were IBS-C; $D=$ subjects included were IBS-D; $M=$ subjects included were IBS-M. Each + indicates one study with positive results; each - indicates one study with negative results.

medications and fiber appears to be beneficial for all types of IBS.

\section{Antispasmodics: Peppermint Oil, Dicyclomine, \\ Hyoscyamine}

Antispasmodics used for the treatment of IBS include peppermint oil, dicyclomine, and hyoscyamine. Hyoscyamine should not be confused with hyoscine, commercially available in the USA as scopolamine; scopolamine is not commonly used for IBS and there is no oral form of scopolamine available in the USA, while hyoscyamine is used commonly for the treatment of IBS despite a lack of a placebo-controlled study assessing its efficacy. The antispasmodics are commonly used for the treatment of IBS, largely because of their generally favorable outcomes in clinical studies.

When recommending peppermint oil for IBS, important considerations are product availability and batch-tobatch consistency, as these products are available over- the-counter and are not FDA-regulated. Despite the lack of regulation, peppermint oil has been well studied and found to be generally effective and well tolerated by patients for the treatment of IBS. Peppermint oil can be initiated at $550 \mathrm{mg}$ once daily or $187 \mathrm{mg}$ three times daily. Although peppermint oil may not require dose adjustment, the dosing frequency of three times daily can make adherence challenging for some patients. Furthermore, in clinical trials, the three times daily doses were administered 30 min prior to meals, which may complicate the dosing further for patients. Once initiated, the onset of efficacy may occur as early as week 2 [19], but may be delayed until week 6 [20]. Adverse effects with peppermint oil in the trials were similar to placebo, with 2 cases of heartburn, one of which resulted from a patient chewing the medication [20,21]. In addition to heartburn, adverse effects associated with peppermint oil in the general population included nausea and vomiting. Enteric-coated peppermint oil products may lessen or prevent heart- 
Table 4. Mechanism of action of medications for the treatment of IBS [27, 34, 37, 39, 40, 42, 49, 57 - 61]

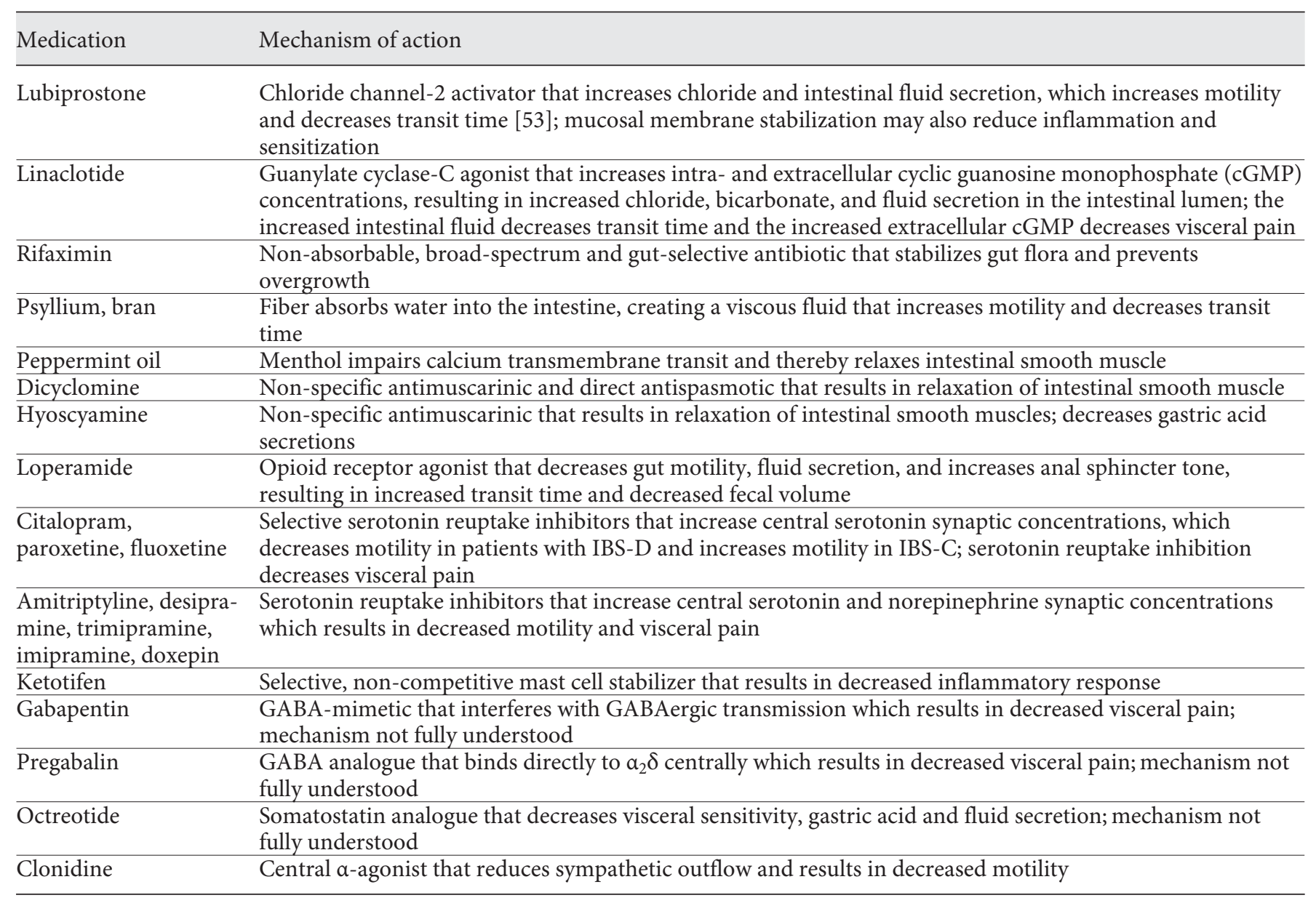

burn. To ensure patients purchase and take the prescribed product and dose, written instructions should be provided by the clinician to the pharmacist, as well as for patients. Detailed instructions for peppermint oil may assist the patient in finding the correct product from among the abundance of over-the-counter products with complicated labels at the pharmacy or health food store.

In contrast to peppermint oil, the antispasmodics of hyoscyamine and dicyclomine are regulated by the FDA and available by prescription, but there is less evidence to support their use when compared to peppermint oil. One clinical trial studied dicyclomine and found favorable outcomes with doses of $40 \mathrm{mg}$ four times daily after 2 weeks of treatment [22]. Although there is no evidence outside of clinical experience to support the use of hyoscyamine, it is commonly used in clinical practice. Frequently used doses of hyoscyamine for the treatment of IBS have ranged from 0.125 to $0.25 \mathrm{mg}$ four times daily and were often used on an as-needed basis. Adverse effects of dicyclomine and hyoscyamine were anticholinergic in nature. Anticholinergic effects reported more frequently with dicyclomine than placebo in the clinical trial included dry mouth, dizziness and blurred vision, which appeared to be dose-related [22]. Other anticholinergic effects to be monitored include constipation, confusion, and falls.

Of the antispasmodics, peppermint oil has the most evidence supporting its use for the treatment of IBS, with limited evidence supporting dicyclomine and no evidence supporting the use of hyoscyamine outside of clinical experience. Although the lack of FDA regulation is limiting, peppermint oil may be a preferred agent for IBS, given the evidence supporting its use and its favorable adverse effect profile. If an alternate antispasmodic is preferred, dicyclomine may be chosen over hyoscyamine given the favorable evidence supporting dicyclomine and its lower cost. 


\section{Loperamide}

Although most commonly used in practice for symptoms of diarrhea, loperamide has been found to be efficacious for multiple symptoms of IBS in persons with all types of IBS. Starting doses of loperamide for IBS have varied from $2 \mathrm{mg}$ at bedtime, $2 \mathrm{mg}$ twice daily and $4 \mathrm{mg}$ at bedtime, with each dosing regimen being well tolerated. Based on the doses found to be efficacious in clinical trials, doses of 3-5 mg daily may be needed to produce symptom relief [23-25], but doses up to $12 \mathrm{mg}$ daily have been safely tolerated [23]. Although the efficacy of loperamide given on an as-needed basis for the treatment of IBS has not been assessed, this is a common practice for managing IBS symptoms of diarrhea with loperamide. Onset of efficacy was apparent in clinical trials beginning at week 3 for some symptoms, but the full benefit of loperamide may not be apparent until week 5 of treatment [24]. Therefore, before treatment failure with loperamide is declared, loperamide should be continued for at least 5 weeks at the doses found to be most commonly effective in clinical trials, 3-5 mg daily. Studies assessing loperamide for the treatment of IBS found adverse effects to be similar when compared to placebo; however, common adverse effects of loperamide observed in the general population included nausea, cramping, and constipation [26].

Advantages of loperamide include its low cost, ease of access for patients as an over-the-counter medication, and long history of use. Additional studies with larger sample sizes, using as-needed dosing regimens, and in specific types of IBS patients are needed to better determine the specific role of loperamide for IBS.

\section{Selective Serotonin Reuptake Inhibitors: Citalopram, Fluoxetine, Paroxetine}

The efficacy data for the selective serotonin reuptake inhibitors (SSRIs), specifically citalopram, fluoxetine and paroxetine, have been conflicting, but generally favorable.

Citalopram has been found to be efficacious for treating IBS when started at $20 \mathrm{mg}$ daily, titrated up to the maintenance dose of $40 \mathrm{mg}$ once daily after 3 weeks [27]. Onset of efficacy was observed beginning at week 3 for some symptoms, but the full effect was not apparent until week 6 of treatment [27]. Fluoxetine improved IBS symptoms at doses of $20 \mathrm{mg}$ once daily and required no titration [28]. Full efficacy was evident as early as week 4 and efficacy persisted even 4 weeks after treatment ended [28]. Similar to fluoxetine, paroxetine also demonstrated positive outcomes. Starting doses for paroxetine were
$12.5 \mathrm{mg}$ once daily, titrated as tolerated to a maximum dose of $50 \mathrm{mg}$ daily [29]. The average dose reached in the trial was $30 \mathrm{mg}$ daily [29], suggesting the most efficacious and tolerable dose was $30 \mathrm{mg}$ daily. Onset of efficacy has not been assessed prior to week 12 [29]. If an SSRI is chosen, treatment should not be discontinued due to lack of efficacy until 4 or 6 weeks for fluoxetine and citalopram, respectively, and perhaps 12 weeks of treatment with paroxetine.

The use of an SSRI for IBS may be especially advantageous for a patient with comorbid depression or anxiety, given SSRIs are highly effective for treating depression and anxiety. Although the adverse effects associated with any of the SSRIs studied for IBS were found to be no different than placebo in the clinical trials, each agent carries the risk of sexual dysfunction, sleep disturbance, serotonin syndrome and weight gain, as seen in the general population. Given the somewhat conflicting evidence supporting the use of the SSRIs, they are generally initiated after other treatment options have failed.

Tricyclic Antidepressants: Amitriptyline, Desipramine, Trimipramine, Imipramine, Doxepin

The tricyclic antidepressants, including amitriptyline, desipramine, trimipramine, imipramine, and doxepin have been studied extensively in the setting of IBS. Imipramine was found to have no benefit, thus management of this agent is not discussed here.

In adults, amitriptyline has demonstrated efficacy at maintenance doses of both $10 \mathrm{mg}$ once [30] and $75 \mathrm{mg}$ at bedtime [31]. Maintenance doses of $75 \mathrm{mg}$ were reached by initiating amitriptyline at $25 \mathrm{mg}$ and titrating up by 25 $\mathrm{mg}$ weekly [31]. Some symptom improvement may be apparent within 4 weeks, but it may take 8 weeks to achieve the maximum benefit of amitriptyline in adults [31]. For adolescents aged 12-18 years, the doses studied and found to be effective were weight-based: $30-50 \mathrm{~kg}$ received $10 \mathrm{mg}$ at bedtime, $50-80 \mathrm{~kg}$ received $20 \mathrm{mg}$ at bedtime, and at least $80 \mathrm{~kg}$ received $30 \mathrm{mg}$ at bedtime [32]. The onset of efficacy for adolescents was not assessed until week 6 [32]; thus it is unclear if the effect occurs earlier than week 6 .

Trimipramine was found to be effective at total daily doses of $50 \mathrm{mg}$, given either once at bedtime [33], or as 10 $\mathrm{mg}$ in the morning and $40 \mathrm{mg}$ at bedtime [34]. Efficacy was not assessed until week 4 of treatment [33], thus it is unknown if the onset of efficacy occurs before week 4 . The only adverse effect reported more frequently than placebo was increased tiredness at night during the first 2 weeks of treatment [34]. 
Desipramine, initiated at $50 \mathrm{mg}$ at bedtime and titrated up by $50 \mathrm{mg}$ weekly to a maintenance dose of $150 \mathrm{mg}$ at bedtime, was found to be efficacious for IBS [35], another study found an initial dose of $150 \mathrm{mg}$ at bedtime to be well tolerated by patients. Statistical significance of adverse effects were not reported, but more patients taking desipramine experienced adverse effects of anxiety, palpitations, sweating and constipation than those taking placebo, which were mostly dose-related [22].

The efficacy of doxepin was also demonstrated in a small study. The doses found to be effective were $75 \mathrm{mg}$ at bedtime and efficacy was determined after 6 weeks of treatment [36].

Advantages of the tricyclics may include their low cost, once-daily dosing, some evidence of efficacy, and many years of experience with their use. Further, patients with concurrent neuropathies, fibromyalgia, recurrent migraines, or psychiatric illness may benefit from treatment with a tricyclic, given the efficacy of tricyclics for these comorbidities. However, adverse effects may limit their use, although these were mostly not apparent in the IBS clinical trials. With the exception of some adverse effects occurring more frequently with trimipramine and desipramine, the occurrence of adverse effects with the tricyclics were no different when compared to placebo in the IBS trials. However, the tricyclics need to be used cautiously, given the common occurrence of adverse effects observed with their use in the general population, which includes anticholinergic effects and QT interval prolongation. Because of their adverse effect profile, the tricyclics are generally reserved for selective patients who have not responded to other treatments.

\section{Ketotifen}

Ketotifen is a new option for treating IBS symptoms. Whereas the oral product studied in clinical trials is not available in the USA, the ophthalmic preparation is commercially available and the oral product may be compounded for a specific patient. Ketotifen's efficacy was observed at maintenance doses of $6 \mathrm{mg}$ twice daily after 8 weeks [37]. It should be initiated at $2 \mathrm{mg}$ twice daily and titrated up by $2 \mathrm{mg}$ twice daily at 2-week intervals [37]. The onset of efficacy was not assessed prior to week 8 of treatment [37]; therefore, efficacy should not be ruled out before week 8 of treatment. The only adverse effect reported to occur more frequently in patients treated with ketotifen than placebo was $2-5 \mathrm{~kg}$ of weight gain [37]. Other adverse effects in the general population have included rash, weight gain, and respiratory infections.
Further studies are needed to define the role of ketotifen for the treatment of IBS, but it may be an option for patients who do not respond to other well-studied medications, especially given its low adverse effect potential.

\section{Anticonvulsants}

Small studies have shown that both pregabalin and gabapentin improve IBS symptoms in persons with all types of IBS and IBS-C, respectively. After a total of 6 days of treatment, gabapentin was found to be efficacious at 200 $\mathrm{mg}$ three times daily after an initial dose of $100 \mathrm{mg}$ three times daily for 3 days [38]. No other IBS treatment has demonstrated onset of efficacy before week 1 of treatment.

The effective maintenance dose of pregabalin was 200 $\mathrm{mg}$ three times daily [39]. Pregabalin was initiated at 50 $\mathrm{mg}$ three times daily for 3 days then titrated up thereafter by $50 \mathrm{mg}$ three times daily every 4 days [39]. Because efficacy was not assessed until week 3 [39], it is unclear whether onset occurs before week 3 of treatment. Therefore, pregabalin should be used for at least 3 weeks.

Adverse effects associated with gabapentin and pregabalin that occurred more frequently than with placebo were dizziness and somnolence [38, 39], which have also been common among the general population taking gabapentin and pregabalin. Given the limited evidence supporting gabapentin and pregabalin for IBS, these agents should be reserved until patients have failed better studied treatments, or perhaps reserved for patients with concomitant neuropathies who may benefit from gabapentin or pregabalin beyond their effects on IBS.

\section{Octreotide}

In contrast to the other IBS treatments, octreotide has required infrequent dosing of every 4 weeks, which is an advantage for some patients. Also unique to octreotide is the intramuscular administration, which may be a deterrent for other patients. One study assessed the impact of octreotide $20 \mathrm{mg}$ intramuscularly every 4 weeks on IBS$\mathrm{M}$ and IBS-C and found some improvement after 8 weeks; however, efficacy was not reported prior to week 8 [40]. Thus, octreotide should be utilized for at least 8 weeks prior to discontinuation. Adverse effects were not reported [40], but patients should be counseled on the risks of use found in the general population. These have included bradycardia, chest pain, fatigue, headache, dizziness, hyperglycemia, abdominal pain, nausea, respiratory infection, and myalgia [41]. Until additional studies of longer duration and larger sample sizes are available, octreotide should be reserved as a last-line option for IBS for patients with severe, refractory symptoms. 


\section{Clonidine}

The antihypertensive clonidine has been found to be effective for some IBS symptoms at $0.1 \mathrm{mg}$ twice daily [42]. The onset of efficacy was not reported, but was apparent after 4 weeks of treatment [42]. Adverse effects reported more frequently with clonidine compared to placebo were drowsiness, dizziness, and dry mouth [42]. Change in blood pressure measurements were not reported [42], but all patients taking clonidine should be monitored closely for orthostatic hypotension and drops in blood pressure. Given the effect of clonidine on blood pressure, persons with concomitant high blood pressure, or attention deficit disorder and IBS-D may benefit from clonidine, given its positive effects on these disease states. The low cost of clonidine is an advantage; however, the risk of rebound hypertension with non-adherence may be a concern (table 5).

\section{Progress in Research for Investigational Drugs for Irritable Bowel Syndrome}

A number of medications for the treatment of IBS are currently in the research pipeline. Some of these medications are currently available for other indications and others are investigational drugs. Further, there are medications available in certain countries that are effective and used for the treatment of IBS, including ramosetron.

A review of clinicaltrials.gov, using the search terms 'irritable bowel syndrome' and 'IBS', identified 22 investigational drugs for the treatment of IBS with ongoing investigation into their utility as a potential treatment [43]. The investigational drug names, proposed mechanism of action and phase in drug development are listed in table 6 . Further, there are 18 drugs that are currently available in the USA that are being studied in clinical trials listed on clinicaltrials.gov for the indication of IBS [63]. Of these medications, 10 have no prior studies assessing their efficacy for the treatment of IBS, which include mesalamine for IBS-D and IBS-A, duloxetine for IBS-A with comorbid depression, crofelemer for IBS-D, milnacipran for IBS-A, escitalopram for IBS-A, dronabinol for IBS-A, colesevelam for IBS-D, nortriptyline for IBS-A, polyethylene glycol for IBS-C, and mexiletine for IBS-A. The remaining 8 currently available drugs being studied in clinical trials to better define their role in therapy for IBS and include pregabalin, rifaximin, citalopram, doxepin, paroxetine $\mathrm{CR}$, alosetron, tegaserod, and desipramine.

The most promising investigational drugs that have completed phase II clinical trials with published positive
Table 5. Current monthly cost of medications used for the treatment of IBS [62]

\begin{tabular}{ll}
\hline Medication & Monthly cost (USD) \\
\hline Lubiprostone & 329.80 \\
Linaclotide & 255.60 \\
Rifaximin & $570.38-634.31^{*}$ \\
Psyllium and bran & Available over-the-counter; cost highly \\
& variable \\
Peppermint oil & Available over-the-counter; cost highly \\
& variable \\
Dicyclomine & 31.66 \\
Hyoscyamine & $46.44-92.88$ \\
Loperamide & Available over-the-counter; cost highly \\
& variable \\
Citalopram & $76.68-79.65$ \\
Paroxetine CR & 332.28 \\
Fluoxetine & 156.08 \\
Amitriptyline & $5.41-26.44$ \\
Desipramine & 184.76 \\
Trimipramine & 174.07 \\
Imipramine & 36.55 \\
Doxepin & 37.29 \\
Ketotifen & Not commercially available; cost variable \\
Gabapentin & due to compounding \\
Pregabalin & 95.83 \\
Octreotide & 395.59 \\
Clonidine & $3,207.67$ \\
& 15.54 \\
\end{tabular}

Monthly cost based on doses used in studies and least expensive dosage form available per Medi-Span [62].

* Based on 10-day treatment with rifaximin $400 \mathrm{mg}$ TID and 14 day treatment with rifaximin $550 \mathrm{mg}$ BID.

outcomes and without documented discontinuation of investigational efforts include JNJ-27018966 (MuDelta), ROSE-010, AST-120, ibodutant, and asimadoline [43]. The results of these most promising investigational drugs are described here.

JNJ-27018966 is a dual $\mu$-opioid agonist and $\delta$-opioid receptor antagonist with demonstrated benefit in patients with IBS-D. A randomized, controlled, double-blind study compared JNJ-27018966 25, 100, and $200 \mathrm{mg}$ twice daily to placebo in 807 patients with IBS-D. The composite of diarrhea and pain was significantly improved in the JNJ-27018966 25 and $200 \mathrm{mg}$ twice-daily groups compared to placebo $(12,13.8$ and $5.7 \%$, respectively, $\mathrm{p}<0.05$ for both comparisons to placebo) [44].

ROSE-010 is a glucagon-like peptide 1 analogue that was studied in a randomized crossover, placebo-controlled trial of 160 patients with IBS and associated ab- 
Table 6. Investigational drugs for treating IBS listed on clinicaltrials.gov: mechanism and development status [43, 63]

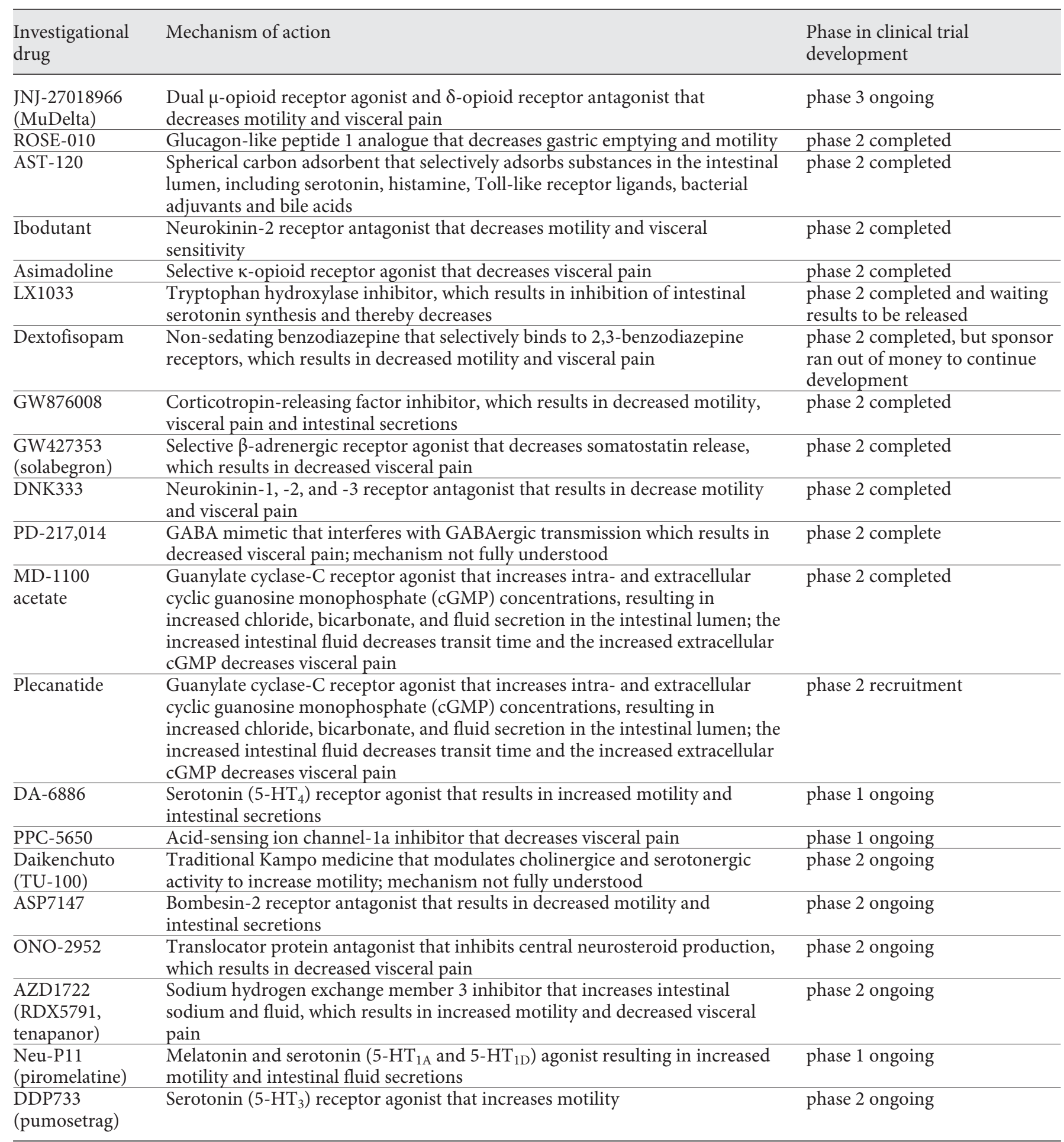


dominal pain. Patients were randomized to ROSE-010 $100 \mu \mathrm{g}$ once daily, $300 \mu \mathrm{g}$ once daily or placebo. Treatment with ROSE-010 resulted in a twofold greater response to abdominal pain compared to placebo ( $\mathrm{p}<0.05$ for all comparisons) and significantly greater patient-reported satisfaction with ROSE-010 ( $\mathrm{p}<0.05)$. The most common treatment-related adverse effect was nausea, which occurred in 19, 37 and $0 \%$ of ROSE-010 $100 \mu \mathrm{g}$, ROSE-010 $300 \mu \mathrm{g}$ and placebo treatments, respectively [45].

AST-120 is a spherical carbon adsorbent originally used to delay renal failure progression and now being studied for its use in the setting of non-constipation-related IBS. A randomized, double-blind, controlled study of 115 non-constipation-related IBS patients demonstrated AST-120 $2 \mathrm{~g}$ three times daily significantly improved the proportion of patients with at least a $50 \%$ reduction in the number of days with abdominal pain compared to placebo (26.8 vs. $10.2 \%$, respectively). Further, AST-120 resulted in significantly improved bloating and numerically improved stool consistency compared to placebo. Adverse effects with AST-120 were similar to placebo [46].

Ibodutant is a neurokinin-2 receptor antagonist that has demonstrated efficacy for the treatment of IBS-D. The results are not published yet, but are available on the clinicaltrials.gov website. In a randomized, double-blind, controlled trial, of 559 IBS-D patients, ibodutant significantly improved abdominal pain, satisfactory relief of overall symptoms, and quality of life compared to placebo. All three doses of ibodutant (1, 3, $10 \mathrm{mg}$ once daily) were superior to placebo, but $10 \mathrm{mg}$ once daily was most effective and females responded better than males [43].

Asimadoline is a $\kappa$-opioid receptor agonist that has demonstrated efficacy in improving IBS symptoms of abdominal pain, urgency and stool frequency. A randomized, controlled, double-blind trial compared asimadoline $0.15,0.5$ and $1 \mathrm{mg}$ twice daily to placebo in $596 \mathrm{pa}$ tients with IBS-D. Asimadoline $0.5 \mathrm{mg}$ twice daily significantly improved by twofold the total number of months with adequate relief of IBS pain, pain scores, urgency and frequency [47].

\section{Conclusion}

The choice of medication therapy for IBS is tailored to the patient's unique symptoms and the evidence supporting the efficacy of a given medication for these symptoms. The abundance of studies assessing medications for IBS are highly variable in quality and the reported efficacy for given IBS symptoms are similarly variable across most studies for a given medication. The quality of available studies and clinical outcomes with medication treatment for IBS can assist the clinician in choosing a medication for a given patient. There are a few medication therapies for IBS with strong evidence demonstrating the safety and efficacy of treatment for IBS, which include lubiprostone, linaclotide, rifaximin, fiber supplementation, and peppermint oil. These agents may be preferred for treating IBS; however, with the exception of peppermint oil, these medications are new and expensive, which may limit their use in practice. Additional well-deigned studies are needed to define optimal dosage regimens of various drugs and to recommend cost-effective treatment strategies for patients with various types of IBS. It is encouraging that there are many ongoing studies of currently available and investigational medications for the treatment of IBS. Of the investigational drugs, JNJ-27018966, a dual $\mu$-opioid agonist and $\delta$-opioid antagonist in phase III clinical trials appear to be most promising as new treatment modalities for IBS.

\section{Disclosure Statement}

The authors have no conflicts of interest to disclose.

References

1 Hungin APS, Chang GR, Locke GR, Dennis $\mathrm{EH}$, Bargout V: Irritable bowel syndrome in the United States: prevalence, symptom patterns and impact. Alimentary Pharmacol Ther 2005;21:1365-1375.

- 2 Brandt LJ, Bjorkman D, Fennerty B, Locke GR, Olden K, Peterson W, Quigley E, Schoenfeld P, Schuster M, Talley N: Systematic review on the management of irritable bowel syndrome in North America. Am J Gastroenterol 2002;97:S7-S26

-3 Brandt LJ, Chey WD, Foxx-Orenstein AE, Quigley EMM, Schiller LR, Schoenfeld PS, Spiegel BM, Talley NJ, Moayyedi P: An evidence-based systematic review on the management of irritable bowel syndrome. Am J Gastroenterol 2009;104:S8-S35.

4 Lubiprostone. Package Insert. Takeda Pharmaceuticals America, Inc., April 2013. Available at: http://www.accessdata.fda.gov/drugsatfda_docs/label/2013/021908s011lbl.pdf. Accessed June 4, 2014.

-5 Johanson JF, Drossman DA, Panas R, Wahle A, Ueno R: Clinical trial: phase 2 study of lubiprostone for irritable bowel syndrome with constipation. Aliment Pharmacol Ther 2008; 27:685-696. 
-6 Johnston JM, Lurtz CB, MacDougall JE, Lavins BJ, Currie MG, Fitch DA, O’Dea C, Baird M, Lembo AJ: Linaclotide improves abdominal pain and bowel habits in a phase IIb study of patients with irritable bowel syndrome with constipation. Gastroenterol 2010; 139:1877-1886.

7 Quigley EMM, Tack J, Chey WD, Rao SS, Fortea J, Falques M, Diaz C, Shiff SJ, Currie MG, Johnston JM: Randomized clinical trials: linaclotide phase 3 studies in IBS-C-a prespecified further analysis based on European Medicines Agency-specified endpoints. Aliment Pharmacol Ther 2013;37:49-61.

-8 Rao SR, Lembo AJ, Shiff SJ, Lavins BJ, Currie MG, Jia XD, Shi K, MacDougall JE, Shao JZ, Eng P, Fox SM, Schneider HA, Kurtz CB, Johnston JM: A 12-week, randomized, controlled trial with a 4-week randomized withdrawal period to evaluate the efficacy and safety of linaclotide in irritable bowel syndrome with constipation. Am J Gastroenterol 2012;107:1714-1724.

-9 Chey WD, Lembo AJ, Lavins BJ, Shiff SJ, Kurtz CB, Currie MG, MacDougall JE, Jia XD, Shao JZ, Fitch DA, Baird MJ, Schneider HA, Johnston JM: Linaclotide for irritable bowel syndrome with constipation: a 26-week, randomized, double-blind, placebo-controlled trial to evaluate efficacy and safety. Am J Gastroenterol 2012;107:1702-1712.

10 Lembo A, Zakko SF, Ferreira NL, Ringel Y, Bortey E, Courtney K, Corsi E, Forbes WP, Pimentel M: Rifaximin for the treatment of diarrhea associated irritable bowel syndrome: short-term treatment leading to long-term sustained response (abstract T1390). Gastroenterology 2008;134(suppl 1):A545.

11 Rifaximin. Package Insert. Salix Pharmaceuticals, Inc., March 2013. Available at: http:// www.accessdata.fda.gov/drugsatfda_docs/ label/2014/021361s013lbl.pdf. Accessed June 4, 2014.

12 Food and Drug Administration. Xifaxan (rifaximin) tablets, $550 \mu \mathrm{g}$ NDA 21-361: Briefing document for gastrointestinal drugs advisory committee meeting 16 November 2011. Available at http://www.fda.gov/downloads/ advisorycommittees/committeesmeetingmaterials/drugs/gastrointestinaldrugsadvisorycommittee/ucm279646.pdf. Accessed September 27, 2013.

13 Jalihal A, Kurian G: Ispaghula therapy in irritable bowel syndrome: improvement in overall well-being is related to reduction in bowel dissatisfaction. J Gastroenterol Hepatol 1990;5:507-513.

14 Bijkerk CJ, de Wit NJ, Muris JW, Whorwell PJ, Knottnerus JA, Hoes AW: Soluble or insoluble fibre in irritable bowel syndrome in primary care? Randomised placebo controlled trial. BMJ 2009;339:b3154.

15 Prior A, Whorwell P: Double blind study of ispaghula in irritable bowel syndrome. Gut 1987;28:1510-1513.
Arthurs Y, Fielding JF: Double blind trial of ispaghula/poloxamer in the irritable bowel syndrome. Irish Med J 1983;76:253.

17 Ritchie JA, Truelove SC: Treatment of irritable bowel syndrome with lorazepam, hyoscine butylbromide, and ispaghula husk. $\mathrm{Br}$ Med J 1979;i:376-378.

18 Longstreth GF, Fox DD, Youkeles L, Forsythe $\mathrm{AB}$, Wolochow DA: Psyllium therapy in the irritable bowel syndrome. Ann Intern Med 1981;95:53-56.

19 Liu JH, Chen GH, Yeh HZ, Huang CK, Poon SK: Enteric-coated peppermint-oil capsules in the treatment of irritable bowel syndrome: a prospective, randomized trial. J Gastroenterol 1997;2:765-768.

20 Alam MS, Roy PK, Miah AR, Mollick SH, Han MR, Mahmud MC, Khatun S: Efficacy of peppermint oil in diarrhea predominant IBS - a double-blind randomized placebo-controlled study. Mymensingh Med 2013;22:27-30.

21 Cappello G, Spezzaferro M, Grossi L, Manzoli L, Marzio L: Peppermint oil (Mintoil) in the treatment of irritable bowel syndrome: a prospective double-blind placebo controlled randomized trial. Dig Liver Dis 2007;39:530536.

22 Page JG, Dirnberger GM: Treatment of the irritable bowel syndrome with Bentyl (dicyclomine hydrochloride). J Clin Gastroenterol 1981;3:153-156.

23 Cann PA, Read NW, Holdsworth CD, Barends D: Role of loperamide and placebo in management of irritable bowel syndrome (IBS). Dig Dis Sci 1984;29:239-247.

24 Efskind PS, Bernklev T, Vatn MH: A doubleblind placebo-controlled trial with loperamide in irritable bowel syndrome. Scand J Gastroenterol 1996;31:463-468.

25 Lavo B, Stenstam M, Nielsen AL: Loperamide in treatment of irritable bowel syndrome - a double-blind placebo-controlled study. Scand J Gastroenterol 1987;130:S77-S80.

26 Loperamide. Package Insert. Janssen Pharmaceutica Inc., July 1998. Available at: http:// www.accessdata.fda.gov/drugsatfda_docs/ label/2005/017694s050lbl.pdf. Accessed June 4, 2014.

27 Tack J, Broekaert D, Fischler B, Van Oudenhove L, Gevers AM, Janssens J: A controlled crossover study of the selective serotonin reuptake inhibitor citalopram in irritable bowel syndrome. Gut 2006;55:1095-1103.

28 Vahedi H, Merat S, Rashidioon A, Ghoddoosi A, Malekzadeh R: The effect of fluoxetine in patients with pain and constipation-predominant irritable bowel syndrome: a doubleblind randomized-controlled study. Aliment Pharmacol Ther 2005;22:381-385.

29 Masand PS, Pae CU, Krulewicz S, Peindl K, Mannelli P, Varia IM, Patkar AA: A doubleblind, randomized, placebo-controlled trial of paroxetine controlled-release in irritable bowel syndrome. Psychosomatics 1990;50: 78-86.
30 Vahedi H, Merat S, Momtahen S, Kazzazi S, Ghaffari N, Olfati G, Malekzadeh R: Clinical trial: the effect of amitriptyline in patients with diarrhoea-predominant irritable bowel syndrome. Aliment Pharmacol Ther 2008;27: 678-684.

31 Rajagopalan M, Kurian G, John J: Symptom relief with amitriptyline in the irritable bowel syndrome. J Gastroenterol Hepatol 1998;13: 738-741.

32 Bahar RJ, Collins BS, Steinmetz B, Ament ME: Double-blind, placebo-controlled trial of amitriptyline for the treatment of irritable bowel syndrome in adolescents. J Pediatr 2008;152: 685-689.

33 Myren J, Groth H, Larssen SE, Larsen S: The effect of trimipramine in patients with the irritable bowel syndrome. Scand J Gastroenterol 1982;17:871-875.

34 Myren J, Lovland B, Larssen S-E, Larsen S: A double-blind study of the effect of trimipramine in patients with irritable bowel syndrome. Scand J Gastroenterol 1984;19:835-843.

-35 Greenbaum DS, Mayle JE, Vanegeren LE, Jerome JA, Mayor JW, Greenbaum RB, Matson RW, Stein GE, Dean HA, Halvorsen NA: Effects of desipramine on irritable bowel syndrome compared with atropine and placebo. Dig Dis Sci 1987;32:257-266.

36 Vij JG, Jiloha RG, Kumar N: Effect of antidepressant drug (doxepin) on irritable bowel syndrome patients. Ind J Psychiatry 1991;33, 243-246.

37 Kooker TK, Braak B, Koopman KE, Welting O, Wouters MM, van der Heide S, Schemann M, Bischoff SC, van den Wijngaard RM, Boeckxstaens GE: The mast cell stabilizer ketotifen decreases visceral hypersensitivity and improves intestinal symptoms in patients with irritable bowel syndrome. Gut 2010;59: 1213-1221.

38 Lee KJ, Kim JH, Cho SW: Gabapentin reduces rectal mechanosensitivity and increases compliance in patients with diarrhea-predominant irritable bowel syndrome. Aliment Pharmacol Ther 2005;22:981-988.

39 Houghton LA, Fell C, Whorwell PJ, Jones I, Sudworth DP, Gale JD: Effect of a second-generation $\alpha_{2} \delta$ ligand (pregabalin) on visceral sensation in hypersensitive patients with irritable bowel syndrome. Gut 2007;56:1218-1225.

40 Klooker TK, Kuiken SD, Lei A, Boeckxstaens GE: Effect of long-term treatment with octreotide on rectal sensitivity in patients with non-constipated irritable bowel syndrome. Aliment Pharmacol Ther 2007;26:605-615.

41 Octreotide. Package Insert. Novartis Pharmaceuticals Corporation March 2012. Available at: http://www.accessdata.fda.gov/drugsatfda_docs/label/2012/019667s061lbl.pdf. Accessed June 4, 2014.

42 Camilleri M, Kim DY, McKinzie S, Kim HJ, Thomforde GM, Burton DD, Low PA, Zinsmeister AR: A randomized, controlled exploratory study of clonidine in diarrhea-predominant irritable bowel syndrome. Clin Gastroenterol Hepatol 2003;1:111-121. 
43 Clinical trials.gov: Find Studies. Available at clinicaltrials.gov. Accessed November 19, 2013.

44 Lazard Capital Markets Annual Healthcare Conference. Furiex Pharmaceuticals. Available at http://files.shareholder.com/downloads / A BEA-4H9PM3/0x 0x550458/ a546b8d0-d614-4136-9514-3c960c74649f/ Lazard\%20Capital\%20Markets\%20Annual\%20Healthcare\%20Conference\%20Presentation. Accessed November 11, 2013.

45 Hellstrom PM, Hein J, Bytzer P, Bjornsson E, Kristensen J, Schambye H: Clinical trial: the glucagon-like peptide- 1 analogue ROSE-010 for management of acute pain in patients with irritable bowel syndrome: a randomized, placebo-controlled, double-blind study. Aliment Pharmacol Ther 2009;29:198-206.

46 Tack JF, Miner PB, Fischer L, Harris MS: Randomised clinical trial: the safety and efficacy of AST-120 in non-constipating irritable bowel syndrome - a double-blind, placebocontrolled study. Aliment Pharmacol Ther 2011;43:868-877.

47 Mangel AW, Bornstein JD, Hamm LR, Buda J, Wang J, Irish W, Urso D: Clinical trial: asimadoline in the treatment of patients with irritable bowel syndrome. Aliment Pharmacol Ther 2008;28:239-249.

48 Whitehead WE, Palsson OS, Gangarosa L, Turner M, Tucker J: Lubiprostone does not influence visceral pain thresholds in patients with irritable bowel syndrome. Neurogastroenterol Motil 2011;23:944-e400.
49 Pimentel M, Sandy P, Mirocha J, Kane SV, Kong Y: The effect of a nonabsorbed oral antibiotic (rifaximin) on the symptoms of the irritable bowel syndrome. Ann Intern Med 2006;145:557-563.

50 Pimental M, Lembo A, Chey WD, Zakko S, Ringel Y, Yu J, Mareya SM, Shaw AL, Bortey E, Forbes WP: Rifaximin therapy for patients with irritable bowel syndrome without constipation. N Engl J Med 2011;364:22-32.

51 Merat S, Khalili S, Mostajabi P, Ghorbani A, Ansari R, Malekzadeh R: The effect of entericcoated, delayed-release peppermint oil on irritable bowel syndrome. Dig Dis Sci 2010;55: 1385-1390.

52 Hovdenak N: Loperamide treatment of the ir ritable bowel syndrome. Scand J Gastroenterol 1987;130:S81-S84.

53 Talley NJ, Kellow JE, Boyce P, Tennant C, Huskic S, Jones M: Antidepressant therapy (imipramine and citalopram) for irritable bowel syndrome: a double-blind, randomized, placebo-controlled trial. Dig Dis Sci 2008;53:108-115.

54 Heefner JD, Wilder RM, Wilson ID: Irritable colon and depression. Psychosomatics 1978; 19:540-547.

55 Ladabaum U, Sharabidze A, Levin TR, Zhao WK, Chung E, Bacchetti P, Jin C, Grimes B, Pepin CJ: Citalopram provides little or no benefit in nondepressed patients with irritable bowel syndrome. Clin Gastroenterol Hepatol 2010;8:42-48.

56 Kuiken SD, Tytgat GNJ, Boeckxstaens GEE: The selective serotonin reuptake inhibitor fluoxetine does not change rectal sensitivity and symptoms in patients with irritable bowel syndrome: a double-blind, randomized, placebo-controlled study. Clin Gastroenterol Hepatol 2003;1:219-228.
57 Forte LR Jr: Uroguanylin and guanylin peptides: pharmacology and experimental therapeutics. Pharmacol Ther 2004;104:137162.

58 Eutamene H, Bradesi S, Larauce M, Theodorou V, Beaufrand C, Ohning G, Fiormonti J, Cohen M, Bryant AP, Kurtz C, Currie MG, Mayer EA, Bueno L: Guanylate cyclase C-mediated antinociceptive effects of linaclotide in rodent models of visceral pain. Neurogastroenterol Motil 2010;22:312-e84.

59 Blikslager AT, Moeser AJ, Gookin JL, Jones SL, Odle J: Restoration of barrier function in injured intestinal mucosa. Physiol Rev 2007; 87:545-564.

60 Hills JM, Aaronson PI: The mechanism of action of peppermint oil on gastrointestinal smooth muscle. Gastroenterology 1991;101: 55-65.

61 Sohn W, Yee OY, Kwon JG, Park KS, Lim YJ Kim TH, Jung SW, Kim JI: Tianeptine vs. amitriptyline for the treatment of irritable bowel syndrome with diarrhea: a multicenter, openlabel, non-inferiority, randomized controlled study. Neurogastroenterol Motil 2012;24: 860-e398.

62 Medi-Span. Medi-Span Average WAC Pricing File. Available at http://www.medispan. com/average-wac-pricing-file/. Accessed November 16, 2013.

63 Bubenik GA: Gastrointestinal melatonin: localization, function, and clinical relevance. Dig Dis Sci 2002;47:2336-2348. 